\title{
THE NEUROLOGICAL ASPECTS OF DERMATOMYOSITIS
}

\section{BY}

\author{
W. B. MATTHEWS and J. C. BURNE
}

\author{
From the Departments of Neurology and Pathology at the Manchester Royal Infirmary
}

Dermatomyositis is a descriptive title for a syndrome first recognized towards the end of the last century by Hepp (1887), Wagner (1887), and Unverricht (1891), and first described in this country by Gowers (1899). The clinical presentation of the condition is extremely variable and when its impact is chiefly on the muscles other muscular and nervous disorders may be simulated. With the exception of Oppenheim (1911), and to a lesser extent of Wilson (1940), dermatomyositis receives scant mention in textbooks of neurology and is seldom discussed in the differential diagnosis of bulbar palsy or of weakness or atrophy of the limbs. It is the purpose of this communication to describe three cases referred for neurological opinion illustrating points of diagnostic difficulty, and to review the subject from a neurological aspect. Some justification may be drawn from the remark of Keil (1940) that " neurologists seem to be hardly familiar with this particular condition".

\section{Case Reports}

Case 1 : N. J. (206663). - A school teacher aged 61 was admitted to the Manchester Royal Infirmary under the care of Dr. F. R. Ferguson on February 9, 1951. She had originally been referred to a chest surgeon on account of persistent fluid at the base of the right lung following a chest infection 18 months earlier. It was noticed that her voice was weak and she also complained of dysphagia. Examination of the larynx showed diminished abduction of the vocal cords, and a barium swallow was thought to suggest a bulbar palsy; the patient was therefore referred for neurological opinion.

The patient said that for the past nine months she had felt increasingly fatigued and weak and had had particular difficulty in preventing her head from dropping forward. Four months ago her voice had become weak and she volunteered the information that this was much more marked towards the end of the day when it became difficult to speak at all. Two months ago she began to have difficulty in swallowing solid foods which seemed to stick at the back of the throat. She lost two stone $(12.7 \mathrm{~kg}$.) in weight and became dyspnoeic on the slightest exertion. To direct questioning she stated that 12 months ago her hands had become swollen, which she attributed to handling Primula japonica. She had some trouble in writing on the blackboard at that time but the swelling soon subsided with resulting improvement in function. She felt, however, that her fingers had never quite recovered their former dexterity. At no time had she experienced any pain and did not complain of any symptoms referable to the skin.

On examination she was seen to be a frail, whitehaired old lady. The optic fundi and pupils were normal. Neither eye could be fully abducted, the right falling short of the outer canthus by $2 \mathrm{~mm}$. and the left by $4 \mathrm{~mm}$., but there was no diplopia. There was slight ptosis of the right eyelid. Sensation on the face was normal and there was no obvious weakness of the facial or masticatory muscles. Her voice was weak and nasal and her cough was extremely weak and ineffective, but the palate and tongue appeared to move normally. The extensor muscles of the neck were weak out of all proportion to her general muscular state. The muscles of the limbs were generally feeble but there was no localized weakness or wasting. There were slight contractures of the flexors of the fingers. The tendon reflexes were all present, the plantars were flexor and there was no sensory loss.

The skin appeared normal except over the hands and wrists. There was some cracking and flaking on the flexor surfaces of the fingers and the skin of the lower forearms seemed somewhat thicker than normal, but there was no erythema. The ankles were oedematous and pitted easily.

There was dullness to percussion at the base of the right lung and the patient became breathless after taking a few steps. The liver and spleen were not palpable and the heart was clinically normal. While under observation she was afebrile.

A blood count showed no abnormality and the erythrocyte sedimentation rate (E.S.R.) was $4 \mathrm{~mm}$. in the first hour (Wintrobe). The blood Wassermann reaction was negative. The basal metabolic rate was increased by $15 \%$. The serum globulin was not raised. A radiograph of the chest showed appearances thought to be those of encysted fluid at the base of the right lung. On screening the upper oesophagus was seen to contain air in the resting state and was quite inert on swallowing. Barium passed through readily in the upright position but not with the patient supine.

The subcutaneous injection of $1.5 \mathrm{mg}$. of neostigmine was without effect on the patient's weakness. A diagnosis 
of dermatomyositis was made, but before further investigation or treatment could be carried out the patient died suddenly of respiratory failure on February 24, 1951.

A post-mortem examination was performed (J. C. B.). The voluntary muscles of the limbs, face, and thoracic and abdominal walls were atrophic, pale, and gelatinous, notably the temporal muscles. The axillary lymph nodes were soft and moderately enlarged. There were thick, fibrous adhesions at the right lung base and there was a grey, fleshy nodule $2 \mathrm{~cm}$. in diameter in the right lateral lobe of the thyroid. No significant abnormality was seen in the other organs. No thymic tissue was seen in the mediastinal fat.

Microscopic Findings.-All sections were stained with haematoxylin and eosin and by the periodic-acid-Schiff technique of McManus (1946) and Hotchkiss (1948) for connective tissue. The muscles examined were the temporal, and the extensors of the forearm, upper oesophagus, diaphragm, and psoas major. All showed oedema with focal lymphocytic and histiocytic infiltration, which was often perivascular. There was atrophy and hyaline degeneration of the muscle fibres and commencing interstitial fibrosis. The muscles of the forearm and oesophagus were only slightly affected, but in the sections of the diaphragm, temporal, and psoas muscles hardly a single healthy muscle bundle was seen (Fig. 1). Despite careful search no evidence of vascular disease was found. Sections of skin from the forearm showed senile elastosis only and the mucosa of the upper oesophagus was normal.

The axillary lymph nodes showed a mild reactive hyperplasia. The right lung base was fibrotic with some mild, non-specific inflammation. The bone marrow appeared normal. The nodule in the thyroid proved to be an early round cell carcinoma but the remainder of the thyroid tissue appeared normal. A microscopic chromophobe adenoma was found in the pituitary. Sections of the spleen, liver, and adrenal, kidney, and

FIG. 1.-Section from diaphragm in Case 1. The muscle bundles are extensively damaged and there is a collection of lymphocytes round a healthy blood vessel. H. and E. $\times 150$.

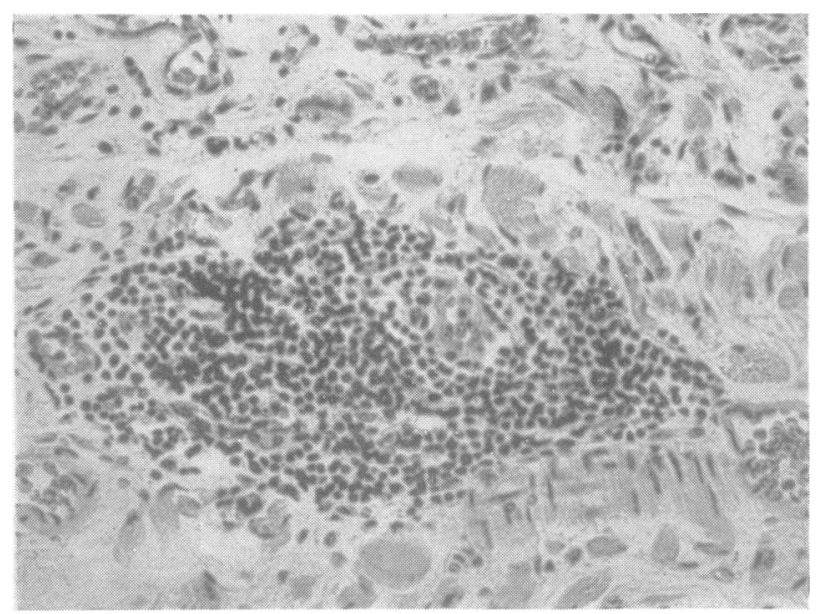

pancreas showed no obvious abnormality. No thymic tissue was found on microscopy.

Important points in the diagnosis were the previous history of swelling of the hands and the slight changes in the skin. The clinical picture otherwise bore a strong resemblance to myasthenia gravis. Some form of thyrotoxic myopathy might be considered in view of the thyroid nodule and of the basal metabolic rate at the upper limit of normal, but is extremely improbable as the carcinomatous cells were undifferentiated and unlikely to show hormonal activity. The pathological changes in the muscles, while extremely striking, cannot be regarded as entirely specific for dermatomyositis.

Case 2 : G. C. (237315). - A woman aged 49 was admitted to the Manchester Royal Infirmary under Dr. F. R. Ferguson in February, 1952, complaining of weakness of the legs and difficulty in swallowing. In November, 1950, she had had a febrile illness lasting a few days with symptoms of malaise and fatigue. These symptoms rapidly passed off, but afterwards she began to notice slowly increasing weakness of the legs, which was especially marked on climbing stairs or on rising from a chair. Eventually the weakness caused great restriction of her activity and she no longer went outside her own house. She thought that her arms $\bigcirc$ were not quite as strong as formerly, but this was only apparent on attempting to lift heavy weights. In May, 1951, she began to have difficulty in swallowing solid foods which seemed to stick high up in the throat. At this point she was admitted to another hospital where oesophagoscopy was performed without any lesion beinge found. It was noted that the eyelids were drooping and that the right side of the face was weak and a diagnosis of myasthenia gravis was made, although there was no response to a test dose of neostigmine. The dysphagia, however, began slowly to improve and by the time she was admitted to the Royal Infirmary she was having little difficulty in swallowing solids, but had noticed that fluids would often regurgitate down her nose. She thought that she could not chew her food as well as formerly and that her voice had become weaker.

On further enquiry she said that two years ago her left ankle had become swollen, but as it was painless she had paid little attention to it. A month before her admission she sustained a minor injury to her right shin and following this her right ankle had also become swollen. For the past year her hands had been liable to go blue in cold weather but had never been swollen. The entire course of her illness had been painless and the most pressing enquiry could not elicit any symptoms referable to the skin. The patient's past health had been good and her last menstrual period had been two years before.

On examination the patient was stout and did not appear ill. There was no abnormality of the optic fundi, pupils, or ocular movements but there was striking bilateral ptosis (Fig. 2). There was no sensory loss on 


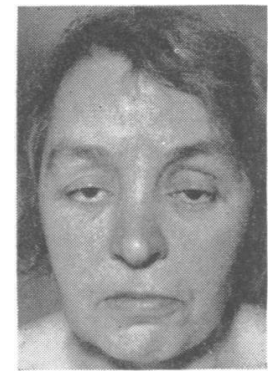

FIG. 2.-Case 2 showing bilateral ptosis. the face and no detectable weakness of the jaw. The right side of the face moved less well than the left. The palate was somewhat pale and oedematous and the right side did not move.

There was no detectable weakness or wasting of the arms but the right biceps and both triceps jerks were absent. There was considerable symmetrical weakness of the muscles of the pelvic girdle, particularly the psoas muscles and glutei. The patient was obese and no wasting could be seen. The consistency of the muscles was not altered neither was there any undue muscle tenderness. The quadriceps muscles were not noticeably weak but the knee jerks were diminished compared with the ankle jerks. The patient walked with the waddling gait of a proximal myopathy and she had great difficulty in rising from a chair. No sensory loss was found and the plantar reflexes were flexor.

The skin over the forehead and cheeks was shiny and somewhat thickened, particularly on the right cheek, and there was some scaling on the forehead. The face and neck would frequently flush in a variable mottled pattern but there was no fixed erythema. The skin around the eyes was of a faintly violaceous hue. These skin changes were only obvious on close inspection and had, indeed, never been noticed by the patient. The skin of the hands was normal. Both feet and ankles were swollen but the appearance of the skin was normal. The swelling was firm and could only be pitted with difficulty and the skin was firmly adherent to the underlying oedematous tissues. The liver and spleen could not be felt and she was afebrile.

A blood count did not show any abnormality but the E.S.R. was $53 \mathrm{~mm}$. in the first hour (Wintrobe). The serum proteins were normal. The cerebrospinal fluid was normal and the Wassermann reaction negative. The urine contained $1,092 \mathrm{mg}$. of creatinine and $239 \mathrm{mg}$. of creatine per 24 hours on a single estimation. A barium swallow showed no constant narrowing of the oesophagus and only a little irregularity of outline. A biopsy taken from the right quadriceps, a muscle not noticeably weak but of easy access, showed no definite abnormality.

She was given $100 \mathrm{mg}$. of adrenocorticotrophic hormone (A.C.T.H.) daily for 10 days. Despite a good eosinopenic response the only change in the clinical condition was a reduction in the violaceous hue of the periorbital skin. The E.S.R. did not fall and there was no significant change in the excretion of creatinine or creatine. In view of the absence of any dramatic change and of the lack of adequate supplies of A.C.T.H. treatment was not further prolonged.

This case had previously been diagnosed as myasthenia gravis. Important points in the history which were not stressed by the patient were the swelling of the ankles and the onset of Raynaud's syndrome. The slight changes in the skin and the oedematous appearance of the palate were significant points in the examination. The negative biopsy from a muscle not grossly weakened in no way affects the diagnosis (Jager and Grossman, 1944).

Case 3 : J. B. (168686). - A girl aged 14 was admitted to the Manchester Royal Infirmary under Dr. F. R. Ferguson on September 1, 1950. She had been born after a normal delivery but her head had been held on one side since birth. Information about her early life was scanty as her mother had died of pulmonary tuberculosis shortly after the child was born. She walked at the age of 2 and did not talk well until she was 4. She attended school but was thought to be backward. At the age of 10 a left sternomastoid tenotomy was done for congenital torticollis with good result, but at about this time the child seemed to stop growing and became progressively weaker and thinner. It was noticed that she had particular difficulty in climbing stairs. This weakness had followed a fluctuating course and had been particularly severe after she had spent three weeks in bed in another hospital. About a year after the onset of the muscular weakness she developed soreness and irritation on the knuckles and later on the face. This skin condition had persisted and had gradually become worse.

The patient had no full-blood siblings, but her stepsister, aged 9, was normal and was already bigger than the patient. No similar disease was known in preceding generations.

On examination the child was small and underdeveloped, being $4 \mathrm{ft}$. (122 cm.) high and weighing only 3 st. (19.1 kg.). There was no mammary development or growth of body hair and she had not menstruated. There was no gross weakness of any muscles supplied by the cranial nerves but the limb musculature was severely and uniformly wasted (Fig. 3). Weakness of the limbs was most marked proximally especially in the legs, leading to great difficulty in climbing stairs and to inability to rise from a supine position. The muscles were not tender but were unduly firm and there were flexion contractures of the elbows and knees. The tendon reflexes were all absent. There was no sensory loss and the plantar reflexes were flexor.

The skin of the face was shiny and atrophic with erythema and slight oedema around the eyes and some

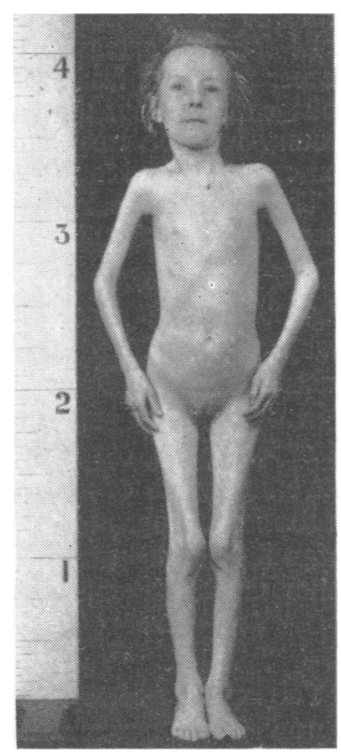

FIG. 3.-Case 3 showing wasting and contractures. 
scaling on the forehead. Over the extensor surfaces of the joints of the upper limbs the skin was atrophic and telangiectatic. On the backs of the thighs there was some faint reticulated pigmentation. The liver and spleen could not be felt and the patient was afebrile.

A blood count showed a mild normochromic anaemia but no other abnormality. The E.S.R. was $43 \mathrm{~mm}$. in the first hour (Wintrobe). The serum proteins, calcium, and phosphorus were within normal limits and a fat balance showed 99\% absorption. Estimation of urinary hormones(Miss B. M. Hain) showed $3 \cdot 1 \mathrm{mg}$. of $17-$ ketosteroids and 3-4 mouse units of folliclestimulating hormone per 24 hours, both values distinctly low for her age. Radiography of the bones showed considerable osteoporosis but normal ossification and dentition for her age. Extensive subcutaneous calcinosis was present (Fig. 4) but no renal calcification.

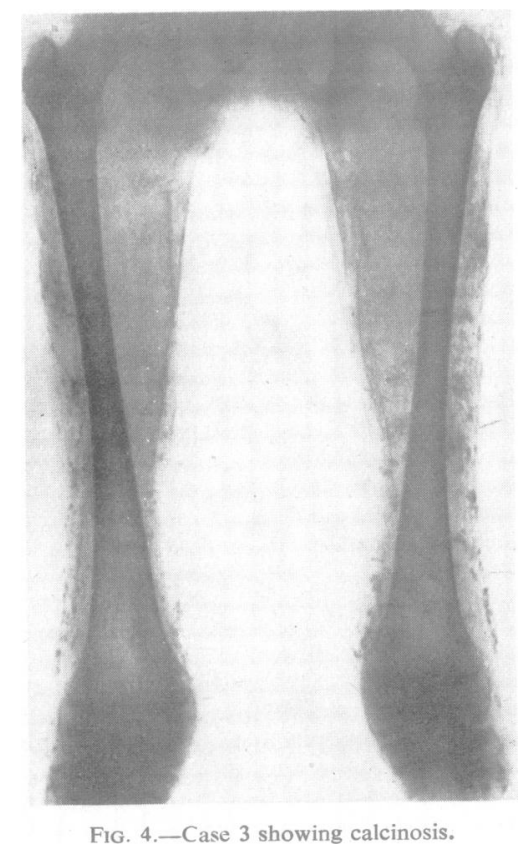

A biopsy was taken from the right quadriceps. At uperation the texture of the skin and of the thin layer of subcutaneous fat appeared normal, but the deep fascia was extremely thick and blended with the underlying pale avascular muscle to form a rigid mass with a gritty feeling to the finger, presumably due to deposits of calcium. Microscopic examination of the fragment removed showed no abnormality of the skin or superficial fat, but extensive calcinosis was present in the deeper fat. The fascia showed slow necrobiosis of the collagenous fibrous tissue and no evidence of an inflammatory reaction was seen. No tissue recognizable as muscle was found in the specimen.

The patient was last seen in December, 1951, when there had been no substantial change in her condition.

Although the cutaneous lesions were much more obvious than in Cases 1 and 2 the correct diagnosis was not established until some four years after the onset, Still's disease and progressive muscular dystrophy being suggested at different stages of the disease. The case is presented as showing the extreme degree of muscular atrophy that can occur in the later stages of chronic dermatomyositis. The infantilism was presumably similar to that seen in other severe chronic diseases in childhood.

\section{Discussion}

The number of reported cases of dermatomyositis is now very large and it is no longer practicable to attempt a comprehensive review. The difficulty of such a task is increased by the unresolved controversy on the specificity of dermatomyositis as a disease and on its possible identity with a group of other conditions including scleroderma and disseminated lupus erythematosus. This problem has been fully reviewed by Banks (1941) and it seems that, while there is clearly an overlap with other " collagen diseases", dermatomyositis retains sufficient individuality to warrant description as a clinical entity.

Onset.-Dermatomyositis is an acute, subacute, or chronic condition of unknown cause affecting both sexes and beginning at any age, even in infancy (Kinney and Maher, 1940), but commonest between the ages of 10 and 50 . The presenting symptoms, which may be preceded by vague prodromata of ill health or by an acute infection, are extremely variable and may be referable to the skin or to the muscles. Steiner (1905) noted that pain in the muscles usually preceded the appearance of a rash, and O'Leary and Waisman (1940) in a review of 40 cases, found that muscular symptoms, either pain or weakness, occurred first in 18 patients. Of Sheard's (1951) 25 cases, muscular fatigue or weakness was the presenting symptom in five, muscular pain in five, and oedema or a rash in 15 . As it is those cases in which muscular weakness is the presenting symptom that are likely to be seen by a neurologist it is important to realize that the interval between the onset of muscular and cutaneous symptoms may be as long as several years (Jager and Grossman, 1944, Case 1). Complaints of dysphagia, dyspnoea, and weakness of the voice may occur early in the disease and accompany those of weakness of the limbs. The onset of Raynaud's syndrome is a not uncommon presenting symptom (Lewis, 1940 ; Dowling, 1940).

Muscular Involvement.-The involvement of the muscles may become widespread, leading to almost complete paralysis of the limbs and trunk (Friedman, 1926), but in less severe cases the weakness of the limbs is often proximal in distribution (Oppenheim, 1911; Barker, 1930) as in Case 2 in the present series. Muscles supplied by the cranial nerves are 
often affected, and diplopia due to weakness of the ocular muscles (O'Leary and Waisman, 1940), nystagmus (Marinesco, Draganesco, and Façon, 1931), ptosis (Kinney and Maher, 1940), facial weakness (O'Leary and Waisman, 1940), weakness of the jaw and tongue (Marinesco and others, 1931), palate (Stuckey, 1935), and neck muscles (Weber and Bode, 1932) have all been reported. Dysphagia is a common symptom occurring in 27 of O'Leary and Waisman's (1940) 40 cases. A weak and nasal voice is often encountered (Carney, 1927) and weakness of the respiratory muscles frequently causes dyspnoea (Kinney and Maher, 1940). According to O'Leary (1949a) the sphincters may be involved. Muscular pain is a common complaint at some stage of the disease but may be absent in the more chronic cases. The muscles are often tender, sometimes to an agonizing degree (Keil, 1940), and muscular oedema is common during acute exacerbations. At this stage the consistency of the muscles may be soft and doughy, while in a later stage they may be unduly hard and no longer tender (Witts, 1936). Atrophy may develop late in the disease and, in addition to the limbs, may involve the tongue (Stuckey, 1935), the masseters (Kinney and Maher, 1940), and sternomastoids (Keil, 1940). When selective the atrophy is usually proximal in distribution but may be distal, and contractures may occur as in Cases 1 and 3 in the present series.

Cutaneous Involvement.-The cutaneous lesions will not be discussed in any detail except to say that they are entirely non-specific and may vary from an erythematous macular rash to frank scleroderma. In the present context it is important to note that such lesions may be insignificant or even entirely absent throughout the course of the disease (Steiner, 1905; O'Leary and Waisman, 1940 ; Sheard, 1951). In other cases they may only occur at certain stages of the disease and may no longer be present when the patient is first seen (Carney, 1927). Nixon (1907) recognized that the muscles might be disproportionately involved and Wilson (1940) also commented on this. Subcutaneous oedema is a very frequent sign and is particularly common in the periorbital tissues. Again the oedema may be present only fleetingly as in the present Case 1.

Systemic Involvement.-Dermatomyositis is not a purely local disease of the skin and muscles, and signs of more widespread lesions may be present. The spleen (Witts, 1936; Ragan, 1950) or liver (Kinney and Maher, 1940) may be enlarged and more rarely there is a generalized lymphadenopathy (de Graciansky, 1949). Articular swelling is another occasional finding. Bruce (1938) reported retinal haemorrhages and exudates in three cases. Fever is common in the acute stages but is far from constant.

Laboratory Diagnosis. - Laboratory aids to diagnosis are sometimes helpful but seldom diagnostic. Schuermann (1939) reviewed the haematological findings which include eosinophilia in less than half the reported cases, and a frequently raised E.S.R. The serum globulin is sometimes markedly increased (Ragan, 1950). As might be expected creatinuria is constantly present (Griffiths, 1940) but the disturbance of carbohydrate metabolism reported by the same author could not be confirmed by Jager and Grossman (1944).

Skin and muscle have frequently been removed at biopsy in an attempt to establish the diagnosis. The findings in the skin are not diagnostic and will not be further discussed. The changes in the muscles have frequently been described but O'Leary (1949b) stated that it had not been possible to recognize a classical histological picture in dermatomyositis. The changes reported in varying degree by many authors consist essentially of atrophy and fragmentation of muscle fibres with an apparent increase in sarcolemma nuclei ; infiltration with lymphocytes, often perivascular; and an increase in interstitial tissue. Dowling (1940) emphasized the lack of specificity of these changes and considered that they closely resembled those found in myasthenia gravis, thyrotoxicosis, and in the myopathy described by Nevin (1936), and Madden (1950) has described similar changes in disseminated lupus erythematosus.

Prognosis.-In most series the mortality is between 50 and $60 \%$, and according to Sheard (1951) the prognosis is worst in acute cases in young adults. Complete recovery is possible but prognosis must be guarded as remissions and exacerbations are commonly reported. Even in apparently "burnt out" cases with severe sclerotic lesions of the muscles there may be some smouldering activity as suggested by the raised E.S.R. in the present Case 3.

Differential Diagnosis.- It is evident that dermatomyositis can present a formidable problem in differential diagnosis. From a neurological point of view most cases fall into one of three groups each with its own diagnostic difficulties. In the first group, of which there is no example in the present series, there is a rapid onset of proximal 
weakness of the limbs with spontaneous pain and increased muscle tenderness and often loss of tendon reflexes. The resemblance to polyneuritis can be striking and cases have been erroneously so diagnosed (Rosenthal and Hoffmann, 1923 ; Keil, 1940). The presence of florid skin lesions and of subcutaneous and muscular oedema usually clarifies the diagnosis, but it is possible that the peripheral nerves may in fact be occasionally involved in dermatomyositis. Senator $(1888,1889,1893)$ diagnosed the condition of "neuromyositis" on the grounds of changes in the nerves and muscles at necropsy, but he could find little clinical evidence of neural involvement beyond tenderness of the nerve trunks and slight sensory changes in one case. Adler (1894) and Levy-Dorn (1895) diagnosed the same combination in their cases. Rosenthal and Hoffmann (1923) considered that loss of tendon reflexes and tenderness of the nerves in their case justified a diagnosis of " dermatoneuromyositis" and Kinney and Maher (1940) also reported demyelination of peripheral nerves. Loss of tendon reflexes, which is of frequent occurrence in dermatomyositis, is not, of course, conclusive evidence of nervous disease, and Oppenheim (1911) stated that reflexes were lost as the particular muscles became involved. Unequivocal signs of disease of the peripheral nerves must be extremely rare but their occasional occurrence may certainly add to the diagnostic difficulties.

In the second group, of which Cases 1 and 2 are examples, there is a more gradual onset of weakness of the limbs accompanied by symptoms suggestive of a bulbar palsy. As has already been emphasized, muscular pain, swelling, or tenderness may be transient or absent and cutaneous lesions may be insignificant. Muscular wasting may suggest a diagnosis of progressive muscular atrophy (Collier and Wilson, 1913 ; Keil, 1940) but the most important source of possible confusion is with myasthenia gravis. Weber and Bode (1932) presented a case of scleroderma combined with myasthenia gravis, a diagnosis which Weber (1938) subsequently amended. In many cases the initial complaint is of easy fatiguability rather than of permanent weakness and the remittant nature of certain symptoms, as with the dysphagia in Case 2 , is another point of similarity with myasthenia gravis. How difficult the distinction may be can be seen from the case of Ragan (1950) in which there appeared to be some response to neostigmine.

In the third group, of which Case 3 is an example, dermatomyositis may simulate other muscular diseases to some degree. Keil (1940) stated that he had known of cases diagnosed as progressive muscular dystrophy but the resemblance can seldom be close. A more probable source of error is the menopausal myopathy of Shy and McEachern (1951a). Before the onset of dysphagia in Case 2 the proximal weakness of the legs in a woman of menopausal age would strongly have suggested this diagnosis. The late myopathy of Nevin (1936) must also be distinguished. The condition of generalized myositis fibrosa (Burton, Cowan, and Miller, 1923 ; Blau, 1938) is probably not a distinct entity. Skin lesions very similar to those of Case 3 were present in both of Blau's cases, and Keil (1940) stated that he had examined one of these patients and considered the diagnosis to be dermatomyositis. In Burton and his colleagues' case transient oedema of the eyelids occurred early in the course of the disease. Calcinosis universalis is also probably a sequel to dermatomyositis in most cases and not a separate entity (Hecht, 1940 ; Nørregaard, 1948).

Treatment.- The correct diagnosis of dermatomyositis is of some practical importance as there have been recent claims of successful treatment. Zarafonetis, Curtis, and Gulick (1950) obtained a good response in one case with para-aminobenzoic? acid, and there have been several claims of success with A.C.T.H. (Oppel, Coker, and Milhorat, 1950 ; Elkinton, Hunt, Godfrey, McCrory, Rogerson, ando Stokes, 1949). Such treatment is not without danger, however, as Ragan (1950) reported an increase in weakness. Shy and McEachern (1951b) have obtained good results with cortisone, especially in acute cases. In assessing the results of treatment the remittant nature of the disease must be remembered, but there seems no doubt that the unknown disease process responsible for the clinical syndrome of dermatomyositis can be influenced by modern methods of treatment.

\section{Summary}

A description is given of three patients with dermatomyositis in whom at one stage alternative diagnoses of muscular or nervous disease were considered. The necropsy findings are given in one case.

The relevant literature is reviewed and it is emphasized that dermatomyositis may present as a variety of syndromes of muscular dysfunction and that the condition must be considered in the differential diagnosis of bulbar palsy, peripheral neuropathy, and the myopathies.

We are grateful to Dr. F. R. Ferguson for permission to report on his patients, and to Professor A. C. P. 
Campbell for advice. We also wish to acknowledge the help of the late Miss B. M. Hain in the hormone assays in Case 3; and of the Department of Medical Photography at the Manchester Royal Infirmary.

\section{REFERENCES}

Adler, - . (1894). Dtsch. med. Wschr., 20, 232.

Banks, B. M. (1941). New Engl. J. Med., 225, 433.

Barker, N. W. (1930). Proc. Mayo Clin., 5, 169.

Blau, A. (1938). J. Mt Sinai Hosp., 5, 432.

Bruce, G. M. (1938). Trans. Amer. ophthal. Soc., 36, 282.

Burton, J. A. G., Cowan, J., and Miller, H. (1923). Quart. J. Med., $17,103$.

Carney, M. J. (1927). Canad. med. Ass. J., 17, ns. 911.

Collier, J., and Wilson, S. A. K. (1913). Proc.roy. Soc. Med., 6 (Neurol. sect.), 119.

Dowling, G. B. (1940). Brit. J. Derm., 52, 242.

Elkinton, J. R., Hunt, A. D., Godfrey, L., McCrory, W. W. Rogerson, A. G., and Stokes, J., Jr. (1949). J. Amer med., Ass., 141, 1273 .

Friedman, E. D. (1926). Med. J. Rec., 123, 382

Graciansky, P. de (1949). Sem. Hôp. Paris, 25, 1406.

Griffiths, W. J. (1940). Brit. J. Derm., 52, 295.

Gowers, W. R. (1899). Brit. med. J., 1, 65.

Hecht, M. S. (1940). J. Pediat., 17, 791

Hepp, P. (1887). Berl. klin. Wschr., 24, 297.

Hotchkiss, R. D. (1948). Arch. Biochem., 16, 131.

Jager, B. V., and Grossman, L. A. (1944). Arch. intern. Med., 73, 271.

Keil, H. (1940). Ibid., 66, 109.

Kinney, T. D., and Maher, M. M. (1940). Amer. J. Path., 16, 561.

Levy-Dorn, M. (1895). Berl. klin. Wschr., 32, 761 .

Lewis, T. (1940). Brit. J. Derm., 52, 233.

McManus, J. F. A. (1946). Nature, 158, 202.
Madden, J. F. (1950). Arch. Derm. Syph., Chicago, 62, 192.

Marinesco, G., Draganesco, S., and Façon, E. (1931). Ann. Méd., 30, 145 .

Nevin, S.'(1936). Quart. J. Med., 5, ns, 51.

Nixon, J. A. (1907). Lancet, $1,79$.

Nørregaard, S. (1948). Acta derm.-venereol., Stockh., 27, 479.

Oppel, T. W., Coker, C., and Milhorat, A. T. (1950). Ann. intern. Med., 32, 318.

Oppenheim, H. (1911). Textbook of Nervous Diseases, 5th ed., trans. Bruce, A. Schulze, Edinburgh.

O'Leary, P. A. (1949a). Med. Clin. N. Amer., 33, 21. Chicago, 59, 362.

- and Waisman, M. (1940). Ibid., 41, 1001.

Ragan, C. (1950). Proceedings of the first clinical A.C.T.H. Conference, Chicago, ed. Mote, J. R., p. 423. Churchill, London.

Rosenthal, C., and Hoffmann, H. (1923). Disch. Z. Nervenheilk. 80,1 .

Senator, H. (1888). Dtsch. med. Wschr., 14, 449.

(1889). Z. klin. Med., 15, 61 .

1893). Dtsch. med. Wschr., 19, 933.

Sheard, C. (1951). Arch. intern. Med., 88, 640

Schuermann, H. (1939). Arch. Derm. Syph., Wien., 178, 414.

Shy, G. M., and McEachern, D. (1951a). Journal of Neurology, Neurosurgery and Psychiatry, 14, ns, 101.

$-(1951 \mathrm{~b})$. Brain, 74, 354.

Steiner, W. R. (1905). J. exp. Med., 6, 407.

Stuckey, E. S. (1935). Brit. J. Derm., 47, 85.

Unverricht, - (1891). Dtsch. med. Wschr., 17, 41.

Wagner, E. (1887). Dtsch. Arch. klin. Med., 40, 241.

Weber, F. Parkes (1938). In discussion on Dowling, G. B. Proc. roy. Soc. Med., 31, 1357.

- -, and Bode, O. B. (1932). Ibid, 25, 966.

Wilson, S. A. Kinnier (1940). Neurology. Arnold, London.

Witts, L. J. (1936). Brit. J. Derm., 48, 509.

Zarafonetis, C. J. D., Curtis, A. C., and Gulick, A. E. (1950). Arch. intern. Med. 85. 27. 